\title{
Diagnostic performance of MRI measurements to assess hindfoot malalignment. An assessment of four measurement techniques
}

Buck, Florian M ; Hoffmann, Adrienne ; Mamisch-Saupe, Nadja ; Farshad, Mazda ; Resnick, Donald ; Espinosa, Norman ; Hodler, Juerg

\begin{abstract}
OBJECTIVE: To investigate the ability of coronal non-weight-bearing MR images to discriminate between normal and abnormal hindfoot alignment. METHODS: Three different measurement techniques (calcaneal axis, medial/lateral calcaneal contour) based on weight-bearing hindfoot alignment radiographs were applied in 49 patients (mean, 48 years; range 21-76 years). Three groups of subjects were enrolled: (1) normal hindfoot alignment $\left(0^{\circ}-10^{\circ}\right.$ valgus); (2) abnormal valgus $\left(>10^{\circ}\right)$; $(3)$ any degree of varus hindfoot alignment. Hindfoot alignment was then measured on coronal MR images using four different measurement techniques (calcaneal axis, medial/lateral calcaneal contour, sustentaculum tangent). ROC analysis was performed to find the MR measurement with the greatest sensitivity and specificity for discrimination between normal and abnormal hindfoot alignment. RESULTS: The most accurate measurement on MR images to detect abnormal hindfoot valgus was the one using the medial calcaneal contour, reaching a sensitivity/specificity of $86 \% / 75 \%$ using a cutoff value of $>11^{\circ}$ valgus. The most accurate measurement on MR images to detect abnormal hindfoot varus was the sustentaculum tangent, reaching a sensitivity/specificity of $91 \% / 71 \%$ using a cutoff value of $<12^{\circ}$ valgus. CONCLUSION: It is possible to suspect abnormal hindfoot alignment on coronal non-weight-bearing MR images. KEY POINTS : • Abnormal hindfoot alignment can be identified on coronal non-weight-bearing MR images. - The sustentaculum tangent was the best predictor of an abnormally varus hindfoot. • The medial calcaneal contour was the best predictor of a valgus hindfoot.
\end{abstract}

DOI: https://doi.org/10.1007/s00330-013-2839-5

Posted at the Zurich Open Repository and Archive, University of Zurich

ZORA URL: https://doi.org/10.5167/uzh-77709

Journal Article

Published Version

Originally published at:

Buck, Florian M; Hoffmann, Adrienne; Mamisch-Saupe, Nadja; Farshad, Mazda; Resnick, Donald; Espinosa, Norman; Hodler, Juerg (2013). Diagnostic performance of MRI measurements to assess hindfoot malalignment. An assessment of four measurement techniques. European Radiology, 23(9):2594-2601. DOI: https://doi.org/10.1007/s00330-013-2839-5 


\title{
Diagnostic performance of MRI measurements to assess hindfoot malalignment. An assessment of four measurement techniques
}

\author{
Florian M. Buck • Adrienne Hoffmann • \\ Nadja Mamisch-Saupe • Mazda Farshad • \\ Donald Resnick • Norman Espinosa • Juerg Hodler
}

Received: 15 November 2012 /Revised: 13 February 2013 / Accepted: 22 February 2013 / Published online: 12 April 2013

(C) European Society of Radiology 2013

\begin{abstract}
Objective To investigate the ability of coronal non-weightbearing MR images to discriminate between normal and abnormal hindfoot alignment.

Methods Three different measurement techniques (calcaneal axis, medial/lateral calcaneal contour) based on weightbearing hindfoot alignment radiographs were applied in 49 patients (mean, 48 years; range 21-76 years). Three groups of subjects were enrolled: (1) normal hindfoot alignment $\left(0^{\circ}-10^{\circ}\right.$

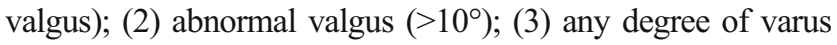
hindfoot alignment. Hindfoot alignment was then measured on coronal MR images using four different measurement techniques (calcaneal axis, medial/lateral calcaneal contour, sustentaculum tangent). ROC analysis was performed to find the MR measurement with the greatest sensitivity and specificity for discrimination between normal and abnormal hindfoot alignment.
\end{abstract}

F. M. Buck $(\bowtie) \cdot$ A. Hoffmann $\cdot$ N. Mamisch-Saupe $\cdot$ J. Hodler Radiology, University Hospital Balgrist \& University of Zurich, Forchstrasse 340,

8008 Zürich, Switzerland

e-mail: florian.buck@balgrist.ch

M. Farshad $\cdot$ N. Espinosa

Department of Orthopaedic Surgery,

University Hospital Balgrist \& University of Zurich,

Forchstrasse 340,

8008 Zürich, Switzerland

D. Resnick

Department of Radiology, University of California San Diego, 3350 La Jolla Village Drive,

San Diego, CA 92161, USA
Results The most accurate measurement on MR images to detect abnormal hindfoot valgus was the one using the medial calcaneal contour, reaching a sensitivity/specificity of $86 \% / 75 \%$ using a cutoff value of $>11^{\circ}$ valgus.

The most accurate measurement on MR images to detect abnormal hindfoot varus was the sustentaculum tangent, reaching a sensitivity/specificity of $91 \% / 71 \%$ using a cutoff value of $<12^{\circ}$ valgus.

Conclusion It is possible to suspect abnormal hindfoot alignment on coronal non-weight-bearing MR images.

Key Points

- Abnormal hindfoot alignment can be identified on coronal non-weight-bearing MR images.

- The sustentaculum tangent was the best predictor of an abnormally varus hindfoot.

- The medial calcaneal contour was the best predictor of a valgus hindfoot.

Keywords Hindfoot alignment · Hindfoot alignment view radiograph $\cdot$ MR imaging $\cdot$ Varus malalignment $\cdot$ Valgus malalignment

\section{Introduction}

Abnormalities of hindfoot alignment are typically found in patients with congenital or acquired hindfoot deformities [1-4]. The recognition of a substantial deviation in hindfoot alignment is important because it influences patient treatment $[5,6]$. A normal hindfoot alignment is defined as a hindfoot valgus angle of $0-5^{\circ}[5]$. An abnormal hindfoot alignment is 
defined as a hindfoot valgus angle greater than $10^{\circ}$ or any degree of hindfoot varus [5]. The relevance of a hindfoot alignment angle of $5-10^{\circ}$ valgus is not clear at this time.

To date, hindfoot alignment has been evaluated on hindfoot alignment radiographs and long axial view radiographs $[7,8]$. Furthermore, many patients with hindfoot alignment deviation undergo magnetic resonance (MR) imaging to assess the tibiotalar and subtalar joints and their supporting structures. Because MR images are acquired under non-weight-bearing conditions, they are not yet considered to be reliable for quantification of hindfoot alignment. However, in the authors' experience, hindfoot alignment deviation can be suspected based on the position of the calcaneus with respect to the tibial shaft in some patients.

Magnetic resonance images in the coronal plane offer numerous anatomical landmarks that could potentially be used for hindfoot alignment measurements such as the medial and lateral contours of the calcaneus and the sustentaculum tali. Therefore, the question arises whether measurements made utilising non-weight-bearing MR images can reliably discriminate between patients with a normal and a clearly abnormal hindfoot alignment. The objective of this study was to investigate this question.

\section{Materials and methods}

Institutional review board approval was obtained, and all patients gave their informed consent to participate in the study. Forty-nine consecutive patients (mean age, 48 years; range, 21-76 years) who had been referred for both MR imaging of the ankle joint and hindfoot alignment radiographs because of clinically suspected hindfoot deformity were included in this prospective study. The cohort was composed of 21 men (mean age, 49 years; range, 2176 years) and 28 women (mean age, 47 years; range, 2768 years). Exclusion criteria were previous fractures of the lower leg or foot, or previous surgery on the ipsilateral lower extremity. No patients had to be excluded from this study.

\section{Hindfoot alignment radiographs}

Upright weight-bearing hindfoot alignment radiographs were acquired using a digital radiography system (Ysio, Siemens Medical Solutions, Erlangen, Germany) as previously described in the literature $[7,8]$. Images were acquired using $70-\mathrm{kV}$ tube voltage and $15-\mathrm{mAs}$ tube current with a source-to-film distance of $150 \mathrm{~cm}$.

Measurements on hindfoot alignment radiographs

Three different techniques were used to measure hindfoot alignment and were employed as independent reference standards (Fig. 1) [7, 9, 10]. This approach was applied because the widely used hindfoot alignment measurement technique, specifically the calcaneal axis, as described by Cobey [7], is prone to measurement errors owing to slight rotation of the foot at the time of image acquisition, whereas measurements using the medial calcaneal contour or the lateral calcaneal contour have proved to be more reliable [9]. The measurements were performed exactly as previously described in the literature $[7,9,10]$ and are illustrated in Fig. 1. A web-based viewing software (ProVision PACS, Release 5.0, Cerner, Kansas City, MO, USA) was used. The measurements were performed independently by two musculoskeletal radiologists with 4 years' (F.M.B.) and 6 years' (N.M.S.) experience in musculoskeletal radiology.

\section{MR imaging}

Magnetic resonance imaging was performed using one of two different 1.5-T (Avanto or Espree, Siemens Medical
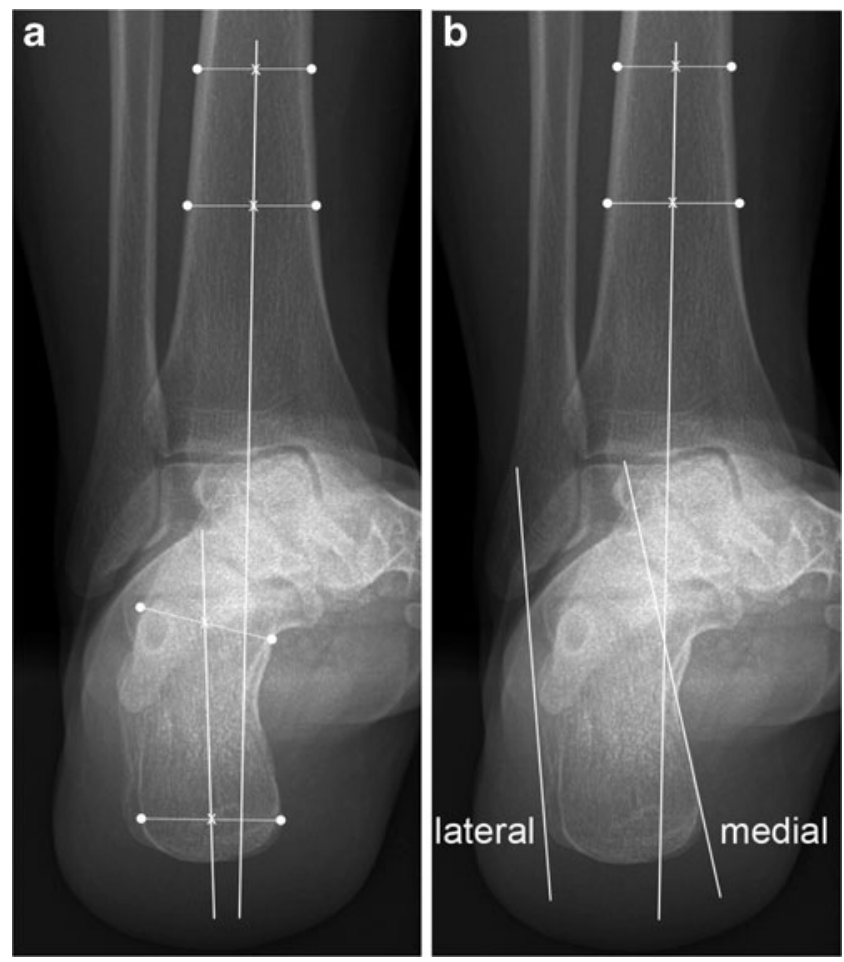

Fig. 1 Measurement of hindfoot alignment on hindfoot alignment radiographs. The axis of the distal tibial shaft served as a reference for all measurements. It was defined by the midpoint of two pairs of points on the cortex of the distal tibia. Hindfoot alignment was measured, as previously described by Cobey [7], between the tibial shaft axis and the calcaneal axis (a). The calcaneal axis was defined by the midpoint between the lateral edge of the calcaneus at the level of the subtalar joint and the corner at the inferior aspect of the sustentacular base and the midpoint between the medial and lateral contours of the posterior calcaneal process. In addition, hindfoot alignment was measured between the tibial shaft axis and adapted lines to the medial and lateral surfaces of the calcaneus as previously described by Donovan and Rosenberg [10] (b) 
Solutions, Erlangen, Germany) or a 3-T MR system (Verio, Siemens Medical Solutions, Erlangen, Germany). A dedicated foot and ankle phased array coil was utilised.

The foot was placed with the plantar surface perpendicular to the tibial shaft axis as in a person standing upright. A standardised examination protocol was acquired in all patients, whereas only a T1-weighted coronal turbo-spin echo sequence (echo time, $23 \mathrm{~ms}$; repetition time, $722 \mathrm{~ms}$; slice thickness, $3 \mathrm{~mm}$; spacing between slices, $3.9 \mathrm{~mm}$; field of view, $160 \times 160 \mathrm{~mm}$; matrix $512 \times 512$; number of excitations, 1; echo train length, 2; flip angle, $140^{\circ}$ ) was used for hindfoot alignment measurements.

\section{Measurements on coronal MR images}

The measurements were performed independently by two radiologists with 3 years (A.H.) and 4 years (F.M.B.) of experience in musculoskeletal radiology. All readers were blinded to the results of the other imaging modality. One reader (F.M.B.) was involved in the measurements on both the hindfoot alignment radiographs and the MR images. To assure blinding for this reader, there was a time interval of at least 1 month between the measurements on hindfoot alignment radiographs and the measurements on MR images. Additionally, the order in which the studies of the patients were reviewed was intentionally not the same in the two readout sessions.

Four different measurements were performed as shown in detail below. All measurements were based on the distal tibial shaft axis. This axis was defined analogously to the tibial shaft axis on the hindfoot alignment radiographs (Figs. 1 and 2a) using the most central coronal image through the distal tibial shaft. This image was identified as the one in which the tibial shaft diameter was maximal, and the tibial cortex was sharply defined (Fig. 2a).

Hindfoot alignment measurement using the calcaneal axis (adapted Cobey technique)

The angle between the tibial shaft axis and the calcaneus axis (adapted Cobey), as shown in Fig. 2, was measured. Analogous to the measurement on hindfoot alignment radiographs, the calcaneal axis was defined by four points on the calcaneal contour. The axis was constructed as a line connecting two points: The first point was the midpoint between the tip of the sustentaculum tali and the superolateral edge of the calcaneus (Fig. 2b); the second point was the midpoint between the medial and lateral contours of the posterior process of the calcaneus on the most posterior image, which included the plantar fascia (Fig. 2b). The tip of the sustentaculum tali was chosen instead of the inferior aspect of the sustentacular base because the latter was not always well defined on the MR images.

Hindfoot alignment measurement using the medial or lateral calcaneal contour

The hindfoot alignment was measured as the angle between the tibial shaft axis and a line adapted to the medial or lateral osseous contour of the calcaneus (Fig. 2c), analogous to the measurements on hindfoot alignment radiographs. These two measurements were also performed on the most
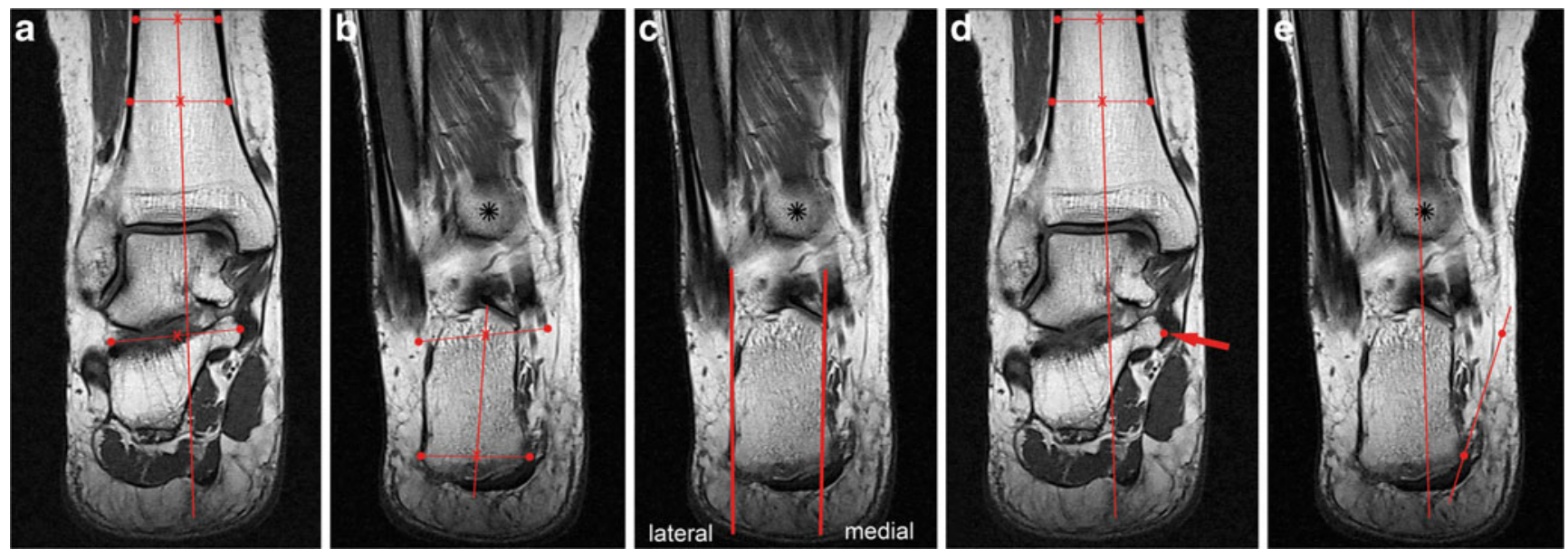

Fig. 2 MR measurement techniques: Angle between the distal tibial shaft (a) and the calcaneal axis (adapted Cobey), which was defined by four points on the calcaneal surface. The axis was defined as (1) the line between the midpoint between the tip of the sustentaculum tali and the superolateral edge of the calcaneus and (2) the midpoint between the medial and lateral contours of the posterior process of the calcaneus on the most posterior image that included the plantar fascia (b). c
Hindfoot alignment axis was measured as the angle between the tibial shaft axis and a line adapted to the medial/lateral surfaces of the calcaneus. $\mathbf{d}$ and $\mathbf{e}$ Angle between the tibial shaft axis and a line drawn at a tangent from the tip of the sustentaculum tali (arrow in d) to the plantar medial surface of the calcaneus (sustentaculum tangent) (e) was measured 
Table 1 Measurement results and interreader agreement

\begin{tabular}{|c|c|c|c|c|c|c|c|}
\hline \multicolumn{2}{|c|}{ Measurement technique } & \multicolumn{2}{|l|}{ Range } & \multicolumn{3}{|l|}{ Average } & \multirow{2}{*}{$\begin{array}{l}\text { Interreader } \\
\text { agreement } \\
\text { Intraclass correlation } \\
\text { coefficient* }\end{array}$} \\
\hline & & $\mathrm{R} 1$ & $\mathrm{R} 2$ & $\mathrm{R} 1$ & $\mathrm{R} 2$ & $\mathrm{R} 1$ and $\mathrm{R} 2$ & \\
\hline \multirow[t]{3}{*}{ Radiographs } & Calcaneal axis (Cobey) & $3-24^{\circ}$ & $4-25^{\circ}$ & $10^{\circ}\left( \pm 0.5^{\circ}\right)$ & $14^{\circ}\left( \pm 0.7^{\circ}\right)$ & $12^{\circ}\left( \pm 0.5^{\circ}\right)$ & 0.69 \\
\hline & Medial calcaneal contour & $0-26^{\circ}$ & $0-36^{\circ}$ & $12^{\circ}\left( \pm 1.0^{\circ}\right)$ & $16^{\circ}\left( \pm 1.3^{\circ}\right)$ & $14^{\circ}\left( \pm 1.0^{\circ}\right)$ & 0.81 \\
\hline & Lateral calcaneal contour & $0-29^{\circ}$ & $0-34^{\circ}$ & $9^{\circ}\left( \pm 1.0^{\circ}\right)$ & $13^{\circ}\left( \pm 1.1^{\circ}\right)$ & $11^{\circ}\left( \pm 1.0^{\circ}\right)$ & 0.82 \\
\hline \multirow[t]{4}{*}{ Coronal MR images } & Calcaneal axis (adapted Cobey) & $0-28^{\circ}$ & $1-31^{\circ}$ & $8^{\circ}\left( \pm 0.8^{\circ}\right)$ & $11^{\circ}\left( \pm 1.0^{\circ}\right)$ & $9^{\circ}\left( \pm 0.9^{\circ}\right)$ & 0.78 \\
\hline & Medial calcaneal contour & $1-29^{\circ}$ & $0-26^{\circ}$ & $9^{\circ}\left( \pm 1.0^{\circ}\right)$ & $10^{\circ}\left( \pm 1.0^{\circ}\right)$ & $10^{\circ}\left( \pm 1.0^{\circ}\right)$ & 0.92 \\
\hline & Lateral calcaneal contour & $1-36^{\circ}$ & $3-32^{\circ}$ & $9^{\circ}\left( \pm 1.0^{\circ}\right)$ & $12^{\circ}\left( \pm 1.0^{\circ}\right)$ & $10^{\circ}\left( \pm 1.0^{\circ}\right)$ & 0.94 \\
\hline & Sustentaculum tangent & $1-35^{\circ}$ & $2-40^{\circ}$ & $17^{\circ}\left( \pm 1.2^{\circ}\right)$ & $20^{\circ}\left( \pm 1.3^{\circ}\right)$ & $18^{\circ}\left( \pm 1.2^{\circ}\right)$ & 0.93 \\
\hline
\end{tabular}

Values in brackets, $95 \%$ confidence interval. *All intraclass correlation coefficients were statistically significant $(P<0.001)$

posterior coronal image, which included the tibia and calcaneus, as previously described by Donovan and Rosenberg [10-12].

Hindfoot alignment measurement using the sustentaculum tangent

The angle between the tibial shaft axis and a tangent drawn between the tip of the sustentaculum tali (Fig. 2d) and the plantar medial osseous contour of the calcaneus (sustentaculum tangent; Fig. 2e) was measured.

\section{Statistical Analysis}

The Pearson correlation coefficient (PCC) was calculated to quantify the correlation between the measurement results on plain films and MR images. Interreader agreement was quantified using the intraclass correlation coefficient (ICC). SPSS (version, 11.5.0; SPSS, Chicago, IL, USA) software was used for PCC and ICC.

Based on the measurements on the hindfoot alignment radiographs, the patients were divided into:

(1) A group with a normal hindfoot alignment $\left(0-5^{\circ}\right.$ valgus $)$ or a hindfoot alignment with questionable relevance (5-10 valgus),

(2) A group consisting of those patients with an abnormal valgus hindfoot alignment $\left(>10^{\circ}\right.$ valgus);
(3) A group consisting of those patients with an abnormal varus hindfoot alignment (any degree of varus). ROC analysis was then performed to find the one best MR measurement to discriminate normal and abnormal varus/valgus hindfoot alignment. Receiver-operating statistics (ROC) was performed by a medical professional with training in advanced statistics (M.F.) to calculate the ability of MR measurements to discriminate between a normal and abnormal valgus and abnormal varus hindfoot alignment as previously defined with respect to the three measurement techniques on hindfoot alignment radiographs. Resulting optimised sensitivity and specificity were calculated. The software Prism (version 4 for Macintosh, Graphpad) was used for ROC analysis.

\section{Results}

According to hindfoot alignment measurements on plain films using the calcaneal axis, a normal hindfoot alignment was found in 24 patients, an abnormal hindfoot valgus alignment was found in 18 patients, and an abnormal hindfoot varus alignment in 7 patients. According to measurements using the medial calcaneal contour, a normal hindfoot alignment was found in 21 patients, abnormal hindfoot valgus alignment was present in 17 patients, and
Table 2 Pearson correlation coefficients of measurements on plain films and MR images

All Pearson correlation coefficients were statistically significant $(P \leq 0.01)$

\begin{tabular}{lcccc}
\hline Measurements on plain films & \multicolumn{2}{l}{ Measurements on MR images } \\
\cline { 2 - 5 } & $\begin{array}{l}\text { Calcaneal axis } \\
\text { (adapted Cobey) }\end{array}$ & $\begin{array}{l}\text { Medial calcaneal } \\
\text { contour }\end{array}$ & $\begin{array}{l}\text { Lateral calcaneal } \\
\text { contour }\end{array}$ & $\begin{array}{l}\text { Sustentaculum } \\
\text { tangent }\end{array}$ \\
\hline Calcaneal axis (Cobey) & 0.57 & 0.56 & 0.51 & 0.57 \\
Medial calcaneal contour & 0.57 & 0.51 & 0.41 & 0.57 \\
Lateral calcaneal contour & 0.53 & 0.61 & 0.49 & 0.47 \\
\hline
\end{tabular}



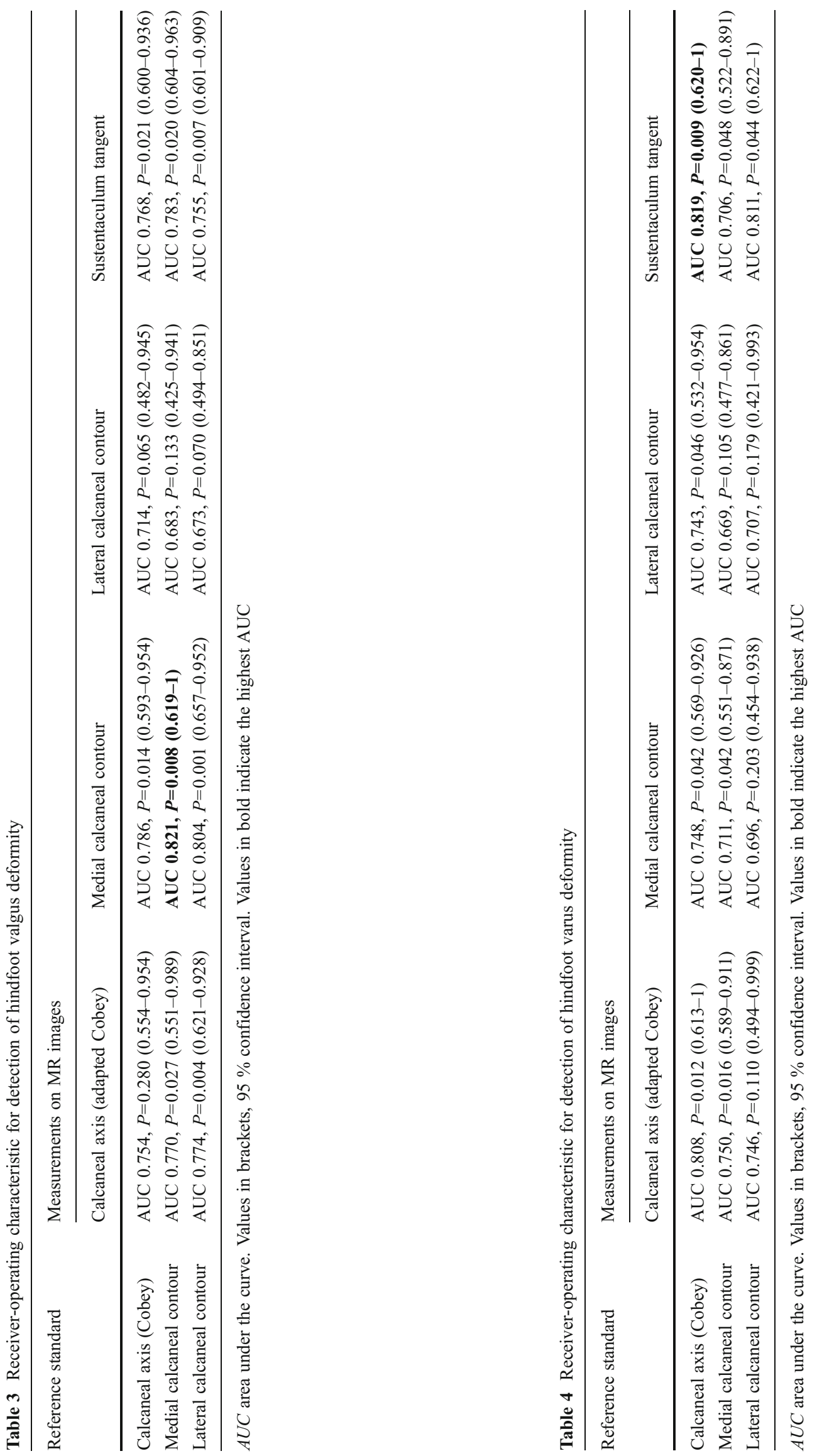


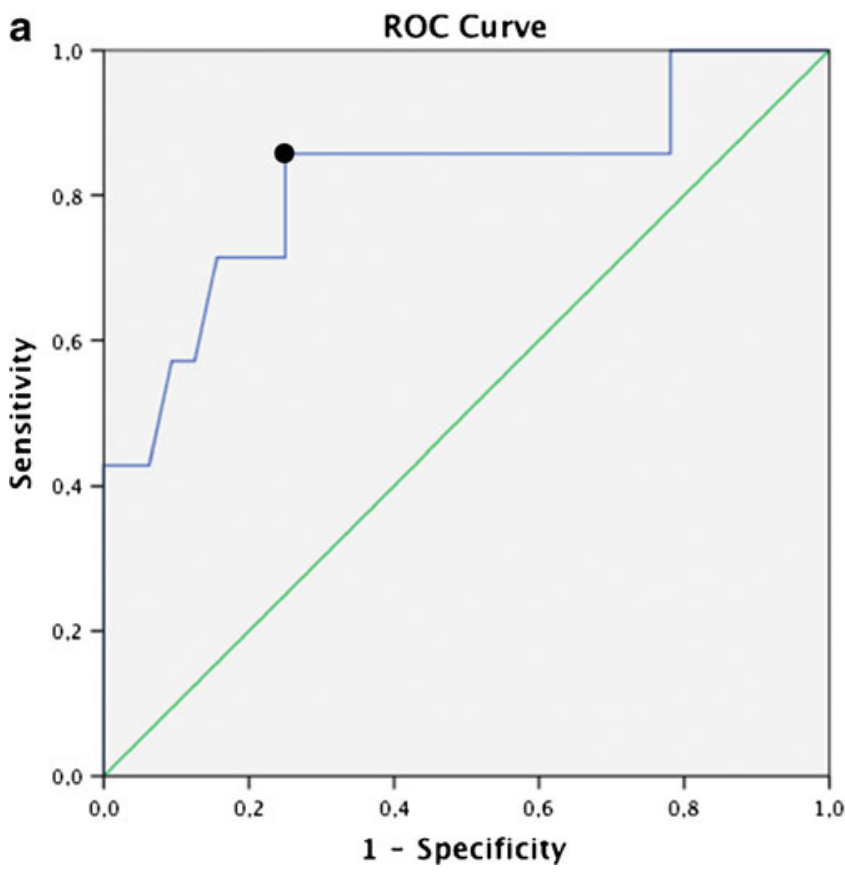

Fig. 3 ROC analysis. Best sensitivity and specificity achievable. a Discrimination of normal hindfoot alignment and abnormal valgus hindfoot alignment by MR measurement using the medial calcaneal contour with respect to measurement technique on plain films also using the medial calcaneal contour. Sensitivity of $86 \%$ and specificity of $75 \%$ were achieved (black dot) by applying a cutoff value of $>11^{\circ}$ valgus. b

abnormal hindfoot varus alignment was found in 11 patients. According to measurements using the lateral calcaneal contour a normal hindfoot alignment was found in 19 patients, abnormal hindfoot valgus alignment was present in 22 patients, and abnormal hindfoot varus alignment was found in 8 patients.

The results of the hindfoot alignment measurements on hindfoot alignment radiographs and coronal MR images are provided in Table 1.

There was a statistically significant $(P \leq 0.01)$ moderate (PCC 0.405-0.614) positive correlation of all measurements on hindfoot alignment radiographs and coronal MR images. The results of Pearson statistics are provided in Table 2.

With respect to discrimination between normal hindfoot alignment and abnormal valgus hindfoot alignment, ROC statistics revealed that MR measurements using the medial calcaneal contour (AUC 0.786-0.821) were the most useful. In terms of discrimination between normal hindfoot alignment and abnormal varus hindfoot alignment, the most statistically significant AUCs were found for MR measurements using the sustentaculum tangent method (AUC 0.706-0.819). Detailed results of ROC statistics are provided in Tables 3 and 4 and Fig. 3.

Concerning the discrimination between normal hindfoot alignment and abnormal hindfoot valgus alignment, the most valuable measurement on MR images with regard to sensitivity

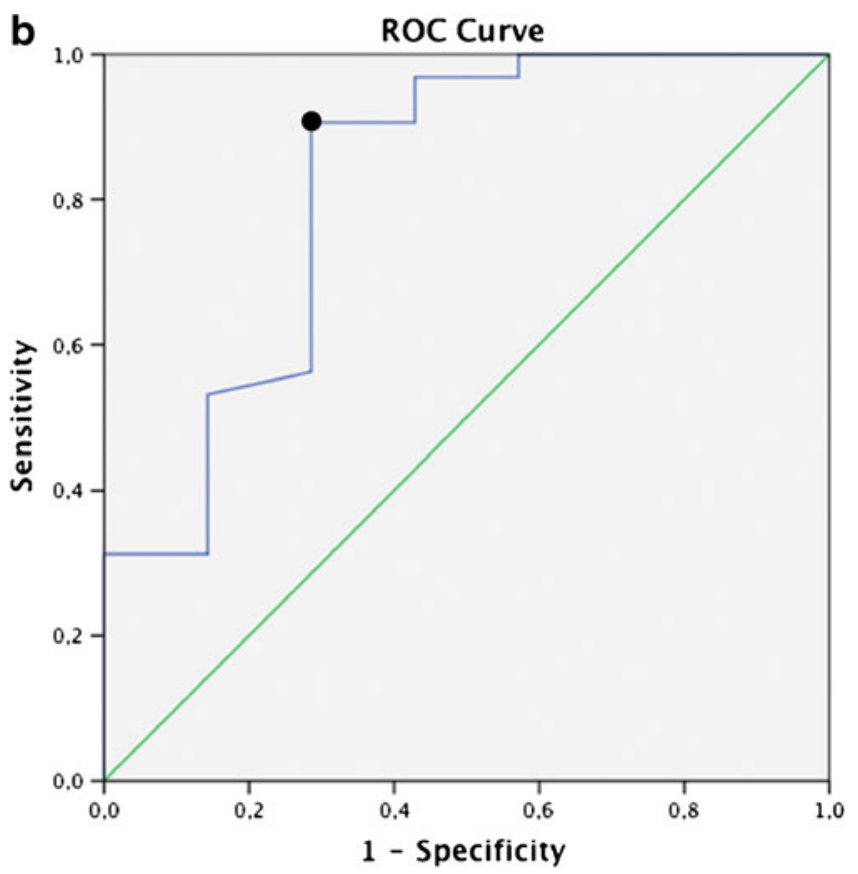

Discrimination of normal hindfoot alignment and abnormal varus hindfoot alignment by MR measurement using the sustentaculum tangent with respect to the measurement technique on plain films using the calcaneal axis. A sensitivity of $91 \%$ and specificity $71 \%$ was achieved (black dot) by applying a cutoff value of $<12^{\circ}$ valgus

and specificity was found to be the one using the medial calcaneal contour, reaching a sensitivity of $86 \%$ and a specificity of $75 \%$ using a cutoff value of $>11^{\circ}$ hindfoot valgus (Fig. 3a, Table 5). Concerning the detection of abnormal hindfoot varus alignment, the most accurate measurement on MR images was found to be the one using the sustentaculum tangent, reaching a sensitivity of $91 \%$ and a specificity of $71 \%$ using a cutoff value of $<12^{\circ}$ valgus (Fig. 3b, Table 5). However, the MR measurement using the medial calcaneal contour also performed well, reaching a sensitivity of $78 \%$ and a specificity of $71 \%$ using a cutoff value of $<6^{\circ}$ valgus (Table 5).

Table 5 Summary of the most valuable measurements on MR images, normal values and their sensitivity and specificity in identifying abnormal hindfoot alignment

\begin{tabular}{llll}
\hline MR measurement using & \multicolumn{2}{l}{ Hindfoot alignment } \\
\cline { 2 - 4 } & $\begin{array}{l}\text { Abnormal } \\
\text { varus }\end{array}$ & Normal & $\begin{array}{l}\text { Abnormal } \\
\text { valgus }\end{array}$ \\
\hline Medial calcaneal contour & $<6^{\circ}$ & & $>11^{\circ}$ \\
Sensitivity & $78 \%$ & $6-11^{\circ}$ & $86 \%$ \\
Specificity & $71 \%$ & & $75 \%$ \\
Sustentaculum tangent & $<12^{\circ}$ & & \\
Sensitivity & $91 \%$ & $\geq 12^{\circ}$ & \\
Specificity & $71 \%$ & & \\
\hline
\end{tabular}


Interreader agreement results (ICC) are included in Table 1 .

\section{Discussion}

Our results demonstrate the ability of measurements made on coronal non-weight-bearing MR images to discriminate between patients with normal hindfoot alignment and patients with abnormal valgus and varus hindfoot alignment.

The highest accuracy for the detection of hindfoot valgus was found using the medial calcaneal contour, reaching a sensitivity of $86 \%$ and a specificity of $75 \%$ using a cutoff value of $>11^{\circ}$ hindfoot valgus. The highest accuracy for detection of hindfoot varus was found using the sustentaculum tangent, reaching a sensitivity of $91 \%$ and a specificity of $71 \%$ using a cutoff value of $<12^{\circ}$ valgus.

Interreader agreement for coronal MR images was higher than for hindfoot alignment radiographs.

Hindfoot alignment measurements are of high clinical relevance. Recently, corrective osteotomies to realign the hindfoot in patients suffering from asymmetric ankle arthritis have been introduced [13-16]. The goal of these procedures is to redirect forces transmitted across the hindfoot and to shift the loading axis into the area of healthy cartilage. In general, planning of any osteotomy is done on conventional x-rays. Therefore, proper assessment of hindfoot axes is crucial. The same applies for other interventions, such as for example total ankle replacement and treatment of any other congenital or acquired hindfoot deformity. The more precise the measurement is, the more accurate the planned corrections will be, which might lead to improved clinical outcomes. The latter remains an issue for future scientific studies.

To the best of our knowledge, there is no method of measuring hindfoot alignment on plain films that meets the demands of perfect interreader agreement and low dependency on the position of the foot at the time of plain film acquisition. Small rotational misplacement of the foot (e.g., $5^{\circ}$ internal rotation) at the time of plain film acquisition can cause a substantial change in hindfoot alignment measurement results [9]. It has been shown that the measurement method using the calcaneal axis is particularly prone to such effects, whereas the measurement methods using the medial or lateral calcaneal contour proved to be more stable [9]. Because the normal range of hindfoot alignment is $10^{\circ}$ or less (neutral, $10^{\circ}$ valgus), rotational misplacement of the foot could have biased our study results. To minimise these effects and because there is no specific, generally accepted and reliable method of measuring hindfoot alignment on plain films, we chose to investigate MR measurements with respect to three different independent standard reference methods on plain films.

In the authors' opinion, MR measurements in addition to measurements on plain radiography are not needed to quantify hindfoot alignment, and MR imaging is certainly not indicated to quantify hindfoot alignment. The purpose of this study was merely to evaluate whether measurements on coronal MR images enable identification of patients with abnormal hindfoot alignment. This aspect is important as MR of the ankle is performed on most of our patients suffering from hindfoot problems.

A recent study by Sutter et al. [17] introduced the possibility of measuring hindfoot alignment on 3D models based on biplanar radiographs. Interreader agreement was considerably better than measurements on plain radiographs. Because this study was performed on human skeleton models and not in patients, this technique still needs to be evaluated in a clinical context.

Despite the quite high sensitivity and specificity of the MR measurements for hindfoot alignment deviation described here, there was no perfect correlation to measurements on radiographs. The reason for this difference is most probably the lack of axial loading of the foot during MR image acquisition. However, this obvious limitation of MR images may not fully explain the difference between the two sets of data. One must expect variation in alignment between weight-bearing and nonweight-bearing images in the subset of patients with subtalar instability due to arthritis, ligament laxity, tendon insufficiency or other causes. In patients with congenital hindfoot alignment deviation with no subtalar joint instability, this restriction may be less pronounced. To the best of our knowledge, there is no definitive report dealing with modifications of hindfoot alignment when the foot is not loaded. To minimise differences, the foot position was always set with the plantar surface perpendicular to the tibial shaft axis, as when standing upright. Robinson et al. [18] compared hindfoot alignment with the neutral and the relaxed calcaneal stance and found a very good correlation between the measurements in the two positions with a constant difference between these measurements. However, a drawback of that study was the inclusion of a potentially heterogeneous patient cohort with little information about the type of suspected hindfoot alignment. The cohort of our study consisted of patients with suspected hindfoot malalignment due to a range of causes encountered at a specialised orthopaedic university clinic. One would expect that MR measurements would perform better in patients with static malalignment and less well in patients with dynamic hindfoot malalignment, but this has to be proven in a future study.

In conclusion, it is possible to suspect abnormal hindfoot alignment on coronal non-weight-bearing MR images.

\section{References}

1. Gibson V, Prieskorn D (2007) The valgus ankle. Foot Ankle Clin 12:15-27 
2. Mendicino RW, Catanzariti AR, Reeves CL, King GL (2005) A systematic approach to evaluation of the rearfoot, ankle, and leg in reconstructive surgery. J Am Podiatr Med Assoc 95:2-12

3. Menz HB (1995) Clinical hindfoot measurement: a critical review of the literature. Foot 5:57-64

4. Strash WW, Berardo P (2004) Radiographic assessment of the hindfoot and ankle. Clin Podiatr Med Surg 21:295-304

5. Coughlin MJ, Mann RA, Saltzman CL (2007) Surgery of the foot and ankle. Mosby, Philadelphia

6. Van Bergeyk AB, Younger A, Carson B (2002) CT analysis of hindfoot alignment in chronic lateral ankle instability. Foot Ankle Int 23:37-42

7. Cobey JC (1976) Posterior roentgenogram of the foot. Clin Orthop Relat Res 118:202-207

8. Reilingh ML, Beimers L, Tuijthof GJ, Stufkens SA, Maas M, van Dijk CN (2010) Measuring hindfoot alignment radiographically: the long axial view is more reliable than the hindfoot alignment view. Skeletal Radiol 39:1103-1108

9. Buck FM, Hoffmann A, Mamisch N, Espinosa N, Resnick D, Hodler J (2011) Hindfoot alignment measurements: rotation-stability of measurement techniques on hindfoot alignment view and long axial view radiographs. AJR Am J Roentgenol 197:578-582

10. Donovan A, Rosenberg ZS (2009) Extraarticular lateral hindfoot impingement with posterior tibial tendon tear: MRI correlation. AJR Am J Roentgenol 193:672-678
11. Saltzman CL, El-Khoury GY (1995) The hindfoot alignment view. Foot Ankle Int 16:572-576

12. Seltzer SE, Weissman BN, Braunstein EM, Adams DF, Thomas WH (1984) Computed tomography of the hindfoot. J Comput Assist Tomogr 8:488-497

13. Tanaka Y, Takakura Y, Hayashi K, Taniguchi A, Kumai T, Sugimoto K (2006) Low tibial osteotomy for varus-type osteoarthritis of the ankle. J Bone Joint Surg Br 88:909-913

14. Takakura Y, Tanaka Y, Kumai T, Tamai S (1995) Low tibial osteotomy for osteoarthritis of the ankle. Results of a new operation in 18 patients. J Bone Joint Surg Br 77:50-54

15. Pagenstert GI, Hintermann B, Barg A, Leumann A, Valderrabano V (2007) Realignment surgery as alternative treatment of varus and valgus ankle osteoarthritis. Clin Orthop Relat Res 462:156168

16. Knupp M, Stufkens SA, Bolliger L, Barg A, Hintermann B (2011) Classification and treatment of supramalleolar deformities. Foot Ankle Int 32:1023-1031

17. Sutter R, Pfirmann CW, Espinosa N, Buck FM (2012) Threedimensional hindfoot alignment measurements based on biplanar radiographs: comparison with standard radiographic measurements. Skeletal Radiol Nov 20. [Epub ahead of print]

18. Robinson I, Dyson R, Halson-Brown S (2001) Reliability of clinical and radiographic measurement of rearfoot alignment in a patient population. Foot 11:2-9 